\title{
Prognostic significance of $\beta$-catenin expression in patients with non-small cell lung cancer: a meta-analysis
}

\author{
Jiajia Jin ${ }^{1 *}$, Ping Zhan ${ }^{2 *}$, Masaru Katoh ${ }^{3}$, Susumu S. Kobayashi ${ }^{4}$, Kevin Phan ${ }^{5}$, Hong Qian ${ }^{1}$, Huijuan Li $^{6}$, \\ Xiaoxia Wang ${ }^{7}$, Xihua Wang ${ }^{8}$, Yong Song ${ }^{2}$; written on behalf of the AME Lung Cancer Collaborative \\ Group
}

${ }^{1}$ Department of Respiratory Medicine, Nanjing General Hospital of Nanjing Military Command, Southeast University, Nanjing 210009, China; ${ }^{2}$ Department of Respiratory Medicine, Jinling Hospital, Nanjing University School of Medicine, Nanjing 210002, China; ${ }^{3}$ Department of Omics Network, National Cancer Center, Tokyo 1040045, Japan; ${ }^{4}$ Lung Cancer Research Program, Beth Israel Deaconess Medical Center, Harvard Medical School, Boston 02215, USA; ${ }^{5}$ The Collaborative Research (CORE) Group, Macquarie University, Sydney, Australia; ${ }^{6}$ Nanjing Medical University, Nanjing 210009, China; ${ }^{7}$ Intensive Care Unit, Inner Mongolia People's Hospital, Hohhot 010017, China; ${ }^{8}$ Department of Respiratory Medicine, Zhongda Hospital, Medical School of Southeast University, Nanjing 210009, China

Contributions: (I) Conception and design: All authors; (II) Administrative support: Y Song, X Wang; (III) Provision of study materials or patients: J Jin, X Wang, Y Song; (IV) Collection and assembly of data: J Jin, P Zhan; (V) Data analysis and interpretation: J Jin, P Zhan; (VI) Manuscript writing: All authors; (VII) Final approval of manuscript: All authors.

*These authors contributed equally to this work.

Correspondence to: Xihua Wang, MD. Department of Respiratory Medicine, Zhongda Hospital, Medical School of Southeast University, \#87, Dingjiaqiao Road, Nanjing 210009, China. Email: wangxihua@126.com; Yong Song, MD, PHD. Department of Respiratory Medicine, Jinling Hospital, Nanjing University School of Medicine, \#305, East Zhongshan Road, Nanjing 210002, China. Email: yong_song6310@yahoo.com.

Background: $\beta$-catenin is a key component of the canonical Wnt pathway, which plays pivotal roles in malignant transformation and cancer progression. Several studies have reported the clinical significance of the expression level of $\beta$-catenin in different subcellular locations. This meta-analysis aimed to assess the prognostic value of $\beta$-catenin expression patterns in patients with non-small cell lung cancer (NSCLC).

Methods: PubMed and Embase databases were searched to identify all articles referring to the association between $\beta$-catenin expression level and outcomes of patients of NSCLC up to November 2016. We included eligible studies to summarize the extracted data in terms of pooled hazard ratios (HRs) and their $95 \%$ confidence intervals (95\% CIs).

Results: A total of 24 studies published between 2000 and 2016 were eligible for this meta-analysis. The total number of patients with NSCLC included was 2,807. Pooled HRs and 95\% CIs suggested that positive $\beta$-catenin expression in membrane was associated with higher survival rates (HR: 0.53; 95\% CI: 0.32-0.87), whereas $\beta$-catenin expression in cytoplasm and nucleus had unfavorable impacts on survival rates with HR of 1.63 (95\% CI: 1.34-1.99) and HR of 3.15 (95\% CI: 1.97-5.05), respectively. But, there was no significant association between $\beta$-catenin expression in abnormal pattern with prognosis (HR: 1.38; 95\% CI: 0.61-3.15). Publication bias was absent in all of the four outcomes. Sensitivity analysis revealed that the results of this meta-analysis were robust.

Conclusions: Reduced membranous $\beta$-catenin, positive expression of cytoplasmic or nuclear $\beta$-catenin is all correlated with poor prognosis, although we did not identify a significant association between abnormal $\beta$-catenin expression and clinical outcome of NSCLC patients. The meta-analysis suggested that membranous, cytoplasmic and nuclear $\beta$-catenin all could serve as an important prognosticator for patients with NSCLC.

Keywords: Meta-analysis; non-small cell lung cancer (NSCLC); precision medicine; genome medicine; prognostic significance; $\beta$-catenin

Submitted Dec 21, 2016. Accepted for publication Feb 07, 2017.

doi: $10.21037 /$ tlcr.2017.02.07

View this article at: http://dx.doi.org/10.21037/tlcr.2017.02.07 


\section{Introduction}

Lung cancer is the leading cause of cancer-related deaths around the world, and nearly $80-85 \%$ of all lung cancers are of the subtype non-small cell lung cancer (NSCLC) (1). Though recent advancements in the diagnosis and treatment have remarkably improved the outcome of patients with NSCLC (2), the prognosis remains poor with an overall 5 -year survival rate of $15 \%(3,4)$. In clinical practice, several independent prognostic factors, disease stage and performance status are important for guiding treatment. However, it is critically important to discover molecular biomarkers and identify precisely the patients at high-risk for poor prognosis (5).

Epithelial-mesenchymal transition (EMT) is a process in which intracellular contacts of epithelial cells are disrupted and intercellular adhesion cells are reduced and epithelial cells acquire migratory fibroblastoid properties (6). EMT is critical for invasive and metastatic progression in cancers (6). EMT is related to reduced epithelial markers, aberrantly increased mesenchymal markers and translocation of $\beta$-catenin in a number of human cancers including gastric cancer (7). $\beta$-catenin could be localized in the membrane, cytoplasm and nucleus of cells (8). $\beta$-catenin exerts dual distinct roles depending on its intracellular localization. $\beta$-catenin expressed on the membrane binds tightly to classical cadherins, regulates cell-to-cell adhesion and cell growth, and has a negative effect on tumor growth (9). Cytoplasmic and nuclear $\beta$-catenin mainly acts as a central molecule in Wingless family (Wnt) signaling pathway (10). In the presence of $\mathrm{Wnt}$ stimulation, the scaffold protein Axin translocates to $\mathrm{Wnt}$ receptor complex and restrains the destruction complex, which allow cytoplasmic $\beta$-catenin to escape degradation and accumulates in the cytoplasm. The elevated free $\beta$-catenin finally translocates to the nucleus where it induces the expression of canonical Wnt target genes, such as CCND1 (Cyclin D1), MYC (c-Myc) and CD44. Wnt signaling is also connected to EMT by upregulating EMT regulators Snail2 (SLUG) and ZEB1 (11). Recently, we have shown that $\beta$-catenin is required for mutant EGFR-induced NSCLC (12). Interestingly, activating of $\beta$-catenin can induce resistance to EGFR tyrosine kinase inhibitors (13). Furthermore, Wnt/ $\beta$-catenin signaling accelerates lung tumorigenesis in vivo (14). Taken together, these findings suggest that $\beta$-catenin is a multifunctional protein and play pivotal roles in malignant transformation, progression and sensitivity to anti-cancer treatments.

It has been widely reported that altered $\beta$-catenin expression is related to poor differentiation, lymph node spread, and metastasis in several human carcinomas, such as gastric cancer (15) and breast cancer (16). In recent years, the association between $\beta$-catenin expression and clinical outcome of patients with NSCLC has been intensively investigated. Yoo et al. (17) reported that positive $\beta$-catenin expression in nucleus or accumulation in cytoplasm in patients with NSCLC was closely associated with shorter overall survival (OS). Whereas, Li et al. (18) showed that reduced level of $\beta$-catenin expression on membrane was related to poor outcomes among NSCLC patients. These facts indicate that there is a controversy on the association between $\beta$-catenin expression and prognosis of NSCLC patients. Here, systematic review and meta-analysis of the current literature will be carried out to obtain a better insight into the connection between $\beta$-catenin expression and survival of NSCLC patients.

\section{Methods}

\section{Search strategy}

The present study follows international guidelines and recommendations on systematic reviews and metaanalyses (19,20). The PubMed and Embase databases were searched using the following mesh terminology: (((((prognostic[Title/Abstract]) OR prognosis[Title/ Abstract]) OR survival[Title/Abstract]) OR outcome[Title/ Abstract])) AND ((NSCLC[Title/Abstract]) OR non-small cell lung cancer[Title/Abstract])) AND ((catenin[Title/ Abstract]) OR beta catenin[Title/Abstract]). The search ended on November 23, 2016. The reference lists associated with the studies were thereafter manually searched. The "Preferred Reporting Items for Systematic Reviews and Meta-Analysis (PRISMA)" guidelines (21) were used.

\section{Inclusion criteria}

Two authors (Jiajia Jin and Ping Zhan) reviewed the studies independently. Studies included in the current metaanalysis had to meet the following criteria: (I) $\beta$-catenin examined by immunohistochemistry and evaluated in human NSCLC tissues; (II) NSCLC patients diagnosed by the golden standard of histopathologic examinations; and (III) sufficient information provided to estimate the hazard ratio (HR) and its $95 \%$ confidence intervals (95\% CIs).

Those articles were excluded: (I) review articles, case reports, letters and laboratory articles; (II) those with duplicate data or overlapping; (III) articles without sufficient data to be extracted. 


\section{Data extraction and quality assessment}

Two reviewers (Jiajia Jin and Ping Zhan) evaluated the eligible studies independently according to the "NewcastleOttawa Scale" (NOS) $(22,23)$. Each of the studies included achieved a score more than five and was rated as qualified. Data retrieved from each report included the first author's name, publication year, study location, histology, metastasis (TNM) stages, the number of patients, antibody sources, the percent of $\beta$-catenin positivity, cut-off value, HR estimate methods, analysis model, HR and $95 \%$ CI, definition of $\beta$-catenin expression, and outcome of patients (Table 1). Patients' outcome was mainly defined as OS and disease free survival (DFS).

\section{Statistical methods}

The HRs and their 95\% CIs were used as the pooled effective value. For some articles not giving HRs and their 95\% CIs explicitly, we extracted the values from KaplanMeier curves as Tierney illustrated previously (39). A statistical test for heterogeneity was performed by the I square test (40). The I square value lies between $0 \%$ to $100 \%$ and it presented with a $95 \% \mathrm{CI}$; studies with I square value over $50 \%$ indicated significant heterogeneity, which required random-effects model (the Mantel-Haenszel method). For studies with I square value less than $50 \%$, the fixed-effects model was used (the DerSimonian and Laird methods). Sensitivity analysis was performed to assess the heterogeneity and robustness of pooled proportions was explored by conducting sensitivity analyses. Moreover, we assessed publication bias through Begg's funnel plots. Meta-analysis was conducted using STATA version 12.0 (Stata Corporation, College Station, TX, USA) statistical software.

\section{Results}

\section{Study selection and characteristics}

The electronic literature search yielded a total of 113 citations eligible for inclusion and the flow diagram of article selection is shown in Figure 1. After reviewing the title and abstract of the articles, 43 articles were excluded. The remaining 70 papers were approved through scrutinizing the entire paper. Of those excluded, one analyzed $\beta$-catenin protein expression level using tumor micro array (TMA) instead of immunohistochemistry method (41); one measured $\beta$-catenin protein expression in adenocarcinoma brain metastases rather than pulmonary tumor tissue (42); three literatures evaluated the prognostic significance of $\beta$-catenin but we could not obtain or calculate the HR value and failed to contact the author (43-45). Additionally, among articles included, one article investigated clinical significance of membranous, cytoplasmic and nuclear $\beta$-catenin staining (18). One article analyzed the association between $\beta$-catenin expression level and NSCLC in different stages (25) and one literature provided information about adenocarcinoma and squamous cell cancer (33). Therefore, we processed these articles as several independent studies in the meta-analysis. Eventually, 17 articles containing 24 studies published between 2000 and 2016 fulfilled the inclusion criteria and were reviewed in our meta-analysis $(17,18,24-38)$, with a total of 2,848 patients with NSCLC enrolled. The major characteristics of the 17 eligible publications are reported in Table 1 . The cases size ranged from 35 to 522 patients. Among the 24 studies, seven focused on membranous $\beta$-catenin $(18,28,30,34,36-38)$, ten focused on cytoplasmic $\beta$-catenin $(18,25-27,33)$, two on nuclear protein $(18,24)$ and five on abnormal $\beta$-catenin expression $(17,29,31,32,35)$.

\section{Impact of $\beta$-catenin expression in membrane on $O S$ of NSCLC in the meta-analysis}

Seven studies assessed the relationship between $\beta$-catenin expression level in membrane and OS of NSCLC patients (Figure 2). The pooled HR was 0.53 (95\% CI: $0.32-0.87$ ) with significant heterogeneity $\left(\mathrm{I}^{2}=76.6 \%, \mathrm{P}<0.001\right)$. This suggests that positive $\beta$-catenin expression in membrane was significantly associated with a better prognosis of NSCLC. To explain the heterogeneity, we conducted the subgroup analysis (Table 2). The results indicated a significant relationship between $\beta$-catenin expression in the membrane and OS was also exhibited in articles using univariate analysis model (HR: 0.38; 95\% CI: 0.24-0.60; $\mathrm{I}^{2}=0.0 \% ; \mathrm{P}=0.863$ ), in articles using calculated HRs (HR: 0.36; 95\% CI: $\left.0.32-0.64 ; \mathrm{I}^{2}=0.0 \% ; \mathrm{P}=0.653\right)$ and in studies with a number of patients less than 100 (HR: $0.41 ; 95 \%$ CI: $\left.0.30-0.57 ; \mathrm{I}^{2}=0.0 \% ; \mathrm{P}=0.907\right)$.

\section{Impact of $\beta$-catenin expression in cytoplasm on clinical outcome of NSCLC in the meta-analysis}

Ten eligible studies investigated the prognostic significance of $\beta$-catenin located in cytoplasm (Figure 3). We found that there was also a significant association between $\beta$-catenin 


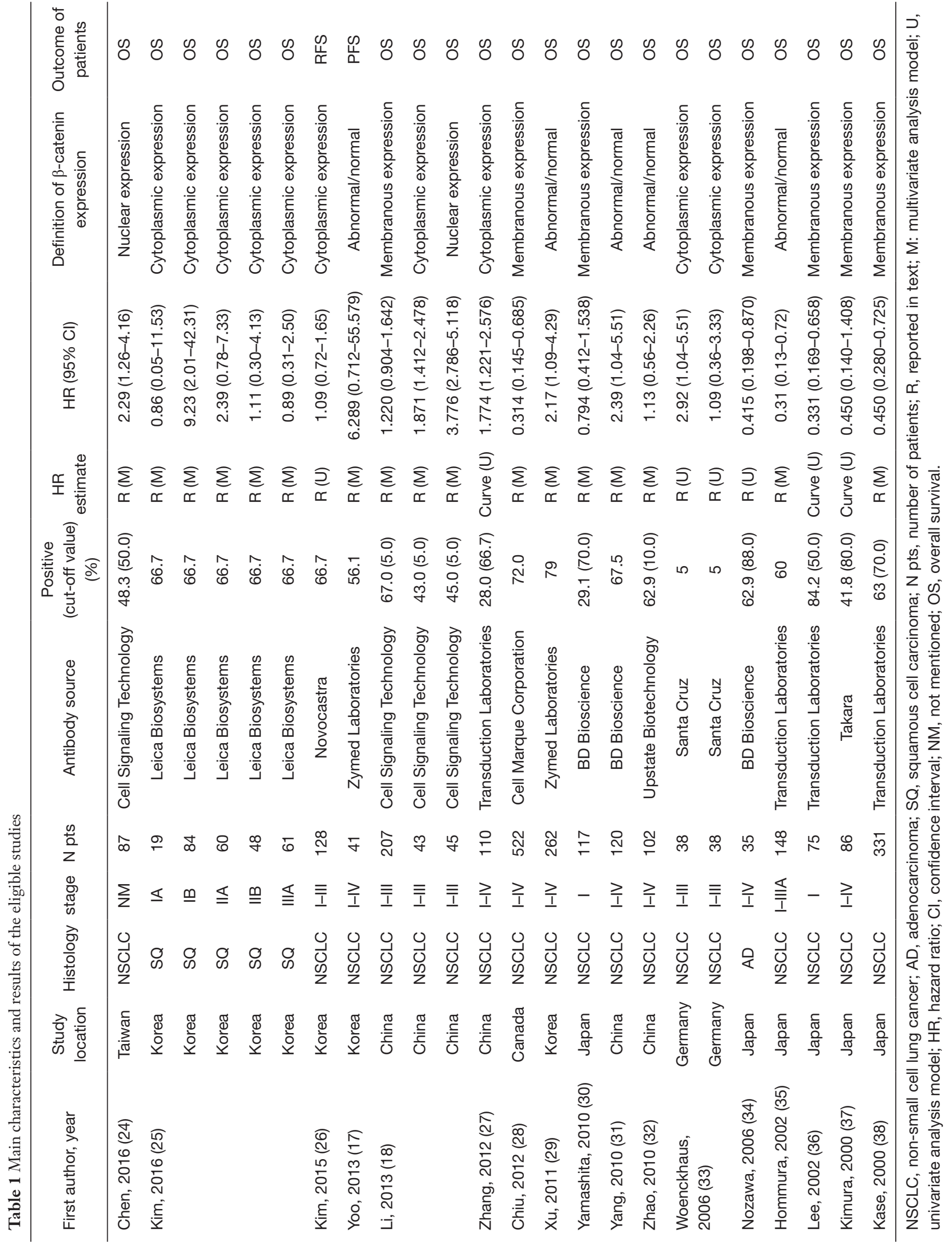




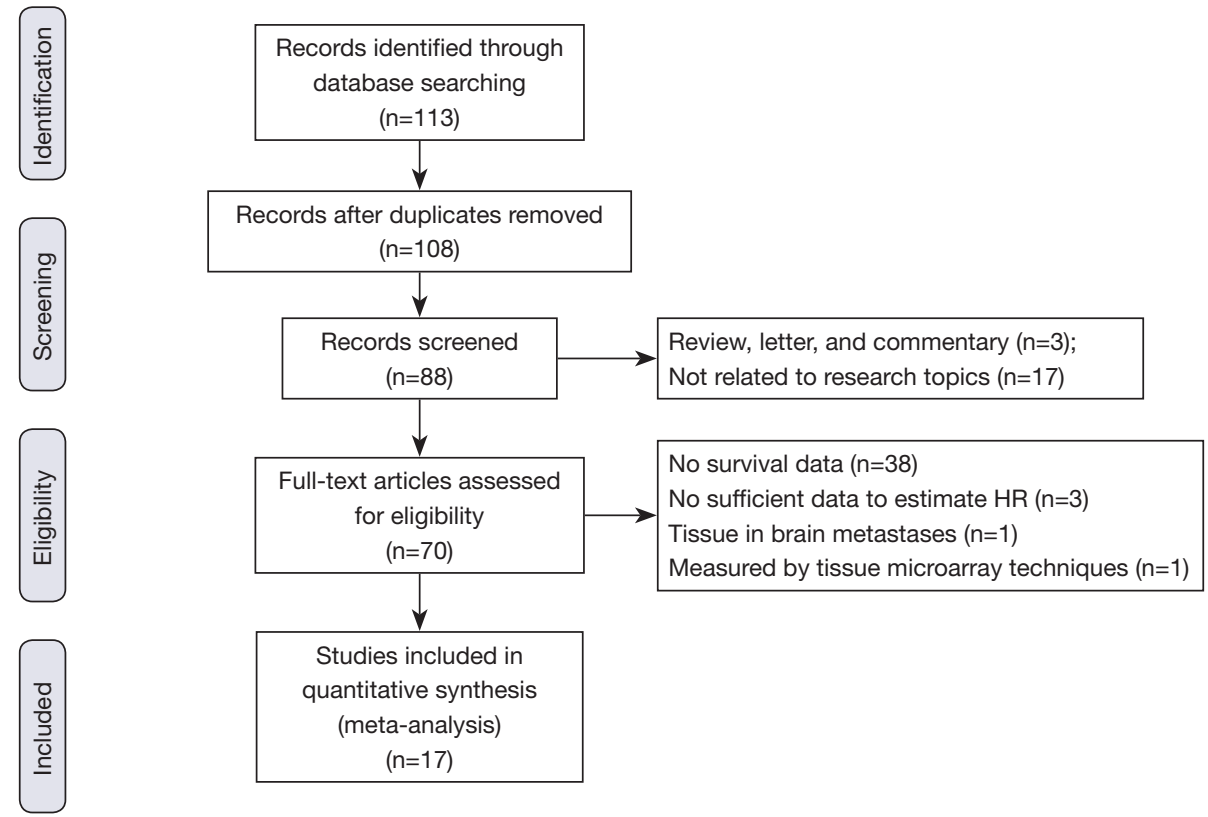

Figure 1 Flow diagram of the study selection in our meta-analysis.

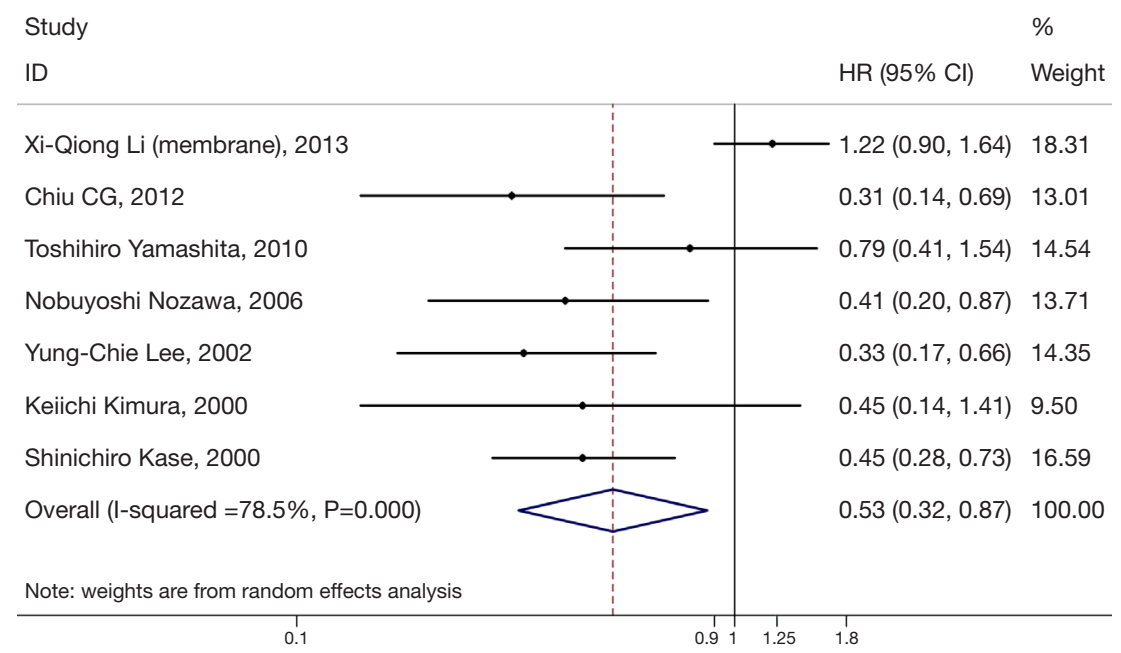

Figure 2 Meta-analysis of the association between positive membranous $\beta$-expression and overall survival in patients with non-small cell lung cancer (NSCLC). Forrest plot of hazard ratios (HRs) and 95\% confidence intervals (CI) from each study were shown.

overexpression in the cytoplasm with worse prognosis; the combined HR was 1.63 (95\% CI: $1.34-1.99)$ with no significant heterogeneity $\left(\mathrm{I}^{2}=36.5 \%, \mathrm{P}=0.117\right)$.

Subgroup analysis was performed by analysis model, study location, number of patients, and methods of HR estimate (Table 2). A significant connection between cytoplasmic $\beta$-catenin expression and clinical outcomes of patients with NSCLC also shown in studies using HR reported in text (HR: 1.64; 95\% CI: 1.33-2.01; $\mathrm{I}^{2}=43.6 \%$; $\mathrm{P}=0.077)$ and in studies enrolling patients more than 100 (HR: 1.62; 95\% CI: 1.32-2.00; $\mathrm{I}^{2}=55.6 \%$; $=0.047$ ). Moreover, other factors including analysis model and study location did not influence the significant prognostic value of cytoplasmic $\beta$-catenin expression. 
Table 2 Subgroup-analysis of the association between $\beta$-catenin expression in different subcellular location and survival

\begin{tabular}{llll}
\hline Subgroup & $\begin{array}{c}\text { No. of } \\
\text { studies }\end{array}$ & $\operatorname{HR}(95 \% \mathrm{Cl})$ & $\frac{\text { Heterogeneity }}{\mathrm{I}^{2}(\%) \quad \mathrm{P}}$ \\
\hline
\end{tabular}

Membrane (18,28,30,34,36-38)

Overall effect
Analysis model
Univariate
Multivariate
HR estimate
Reported in text
By estimate
Patients' number
$<100$
$\geq 100$

Study location

$\begin{array}{lllll}\text { Asia } & 6 & 0.57(0.34-0.97) & 78.7 & 0.000 \\ \text { Non-Asia } & 1 & 0.31(0.14-0.68) & & \\ \text { Cytoplasm }(18,25-27,33) & & & & \end{array}$

$\begin{array}{lllll}\begin{array}{l}\text { Overall effect } \\ \text { Analysis model }\end{array} & 10 & 1.64(1.34-1.99) & 36.5 & 0.116 \\ \text { Univariate } & 4 & 1.35(1.00-1.86) & 36.6 & 0.192 \\ \begin{array}{l}\text { Multivariate } \\ \text { HR estimate }\end{array} & 6 & 1.85(1.43-2.38) & 31.1 & 0.202 \\ \text { Reported in text } & 9 & 1.64(1.33-2.01) & 43.6 & 0.077 \\ \text { By estimate } & 1 & 1.62(0.83-3.17) & & \\ \begin{array}{l}\text { Patients' number } \\ <100\end{array} & 4 & 1.75(0.98-3.12) & 0.0 & 0.412 \\ \geq 100 & 6 & 1.62(1.32-2.00) & 55.6 & 0.047\end{array}$

Study location

$\begin{array}{lllll}\text { Asia } & 8 & 1.60(1.30-1.97) & 40.5 & 0.108 \\ \text { Non-Asia } & 2 & 2.05(1.05-3.99) & 48.2 & 0.165\end{array}$

Abnormal expression $(17,29,31,32,35)$

\begin{tabular}{lllll}
$\begin{array}{l}\text { Overall effect } \\
\text { Patients' number }\end{array}$ & 5 & $1.38(0.61-3.15)$ & & \\
$<100$ & 1 & $6.29(0.71-55.56)$ & & \\
$\geq 100$ & 4 & $1.18(0.50-2.77)$ & 80.1 & 0.002 \\
\hline
\end{tabular}

$\mathrm{Cl}$, confidence interval; $\mathrm{HR}$, hazard ratio.
Impact of nuclear and abnormal $\beta$-catenin expression on clinical outcome of NSCLC in the meta-analysis

$\beta$-catenin overexpression in the nucleus was also associated with poorer OS (HR: 3.15; 95\% CI: $1.97-5.05 ; \mathrm{I}^{2}=53.3 \%$; $\mathrm{P}=0.144$ ), but only following the analysis of two limited studies (Figure 4). However, there was no significant association between abnormal $\beta$-expression and OS of NSCLC patients; the combined HR was 1.38 (95\% CI: $0.61-3.15)$, with significant heterogeneity $\left(\mathrm{I}^{2}=76.7 \%\right.$, $\mathrm{P}=0.002$ ) (Figure 5). Moreover, subgroup analysis indicated that the number of patients did not alter the negative prognostic impact of abnormal $\beta$-catenin expression (Table 2).

\section{Publication bias analysis}

Begger's funnel plot was used to check the publication bias in this meta-analysis (Figure 6). There was no publication bias for all of the four outcomes, as evidenced by the symmetrical pattern of the funnel plots.

\section{Sensitivity analysis}

Sensitivity analysis was conducted, and we found that there is little impact of any single study on the combined HR of the four outcomes, which suggested that the results of the meta-analysis were robust (Figure 7).

\section{Discussion}

We carried out a meta-analyses of $\beta$-catenin expression in NSCLC patients based on 24 studies (Table 1), while Yang et al. (46) recently reported a meta-analyses of expression in NSCLC patients based on 15 studies. Membranous $\beta$-catenin that is directly associated with E-cadherin and $\alpha$-catenin is involved in the stabilization of cell-cell adhesion, whereas cytoplasmic and nuclear $\beta$-catenin are important mediators of the canonical Wnt signaling cascade (47-50). The association between overall $\beta$-catenin expression and prognosis of NSCLC patients is controversial $(17,18)$. Because $\beta$-catenin exerts different effects in different subcellular localization, we carried out meta-analyses according to the subcellular localization of $\beta$-catenin, and found that reduced membranous expression of $\beta$-catenin (Figure 2), overexpression of cytoplasmic $\beta$-catenin (Figure 3) and overexpression of nuclear $\beta$-catenin (Figure 4) are all significantly associated with unfavorable prognosis in patients with NSCLC. 


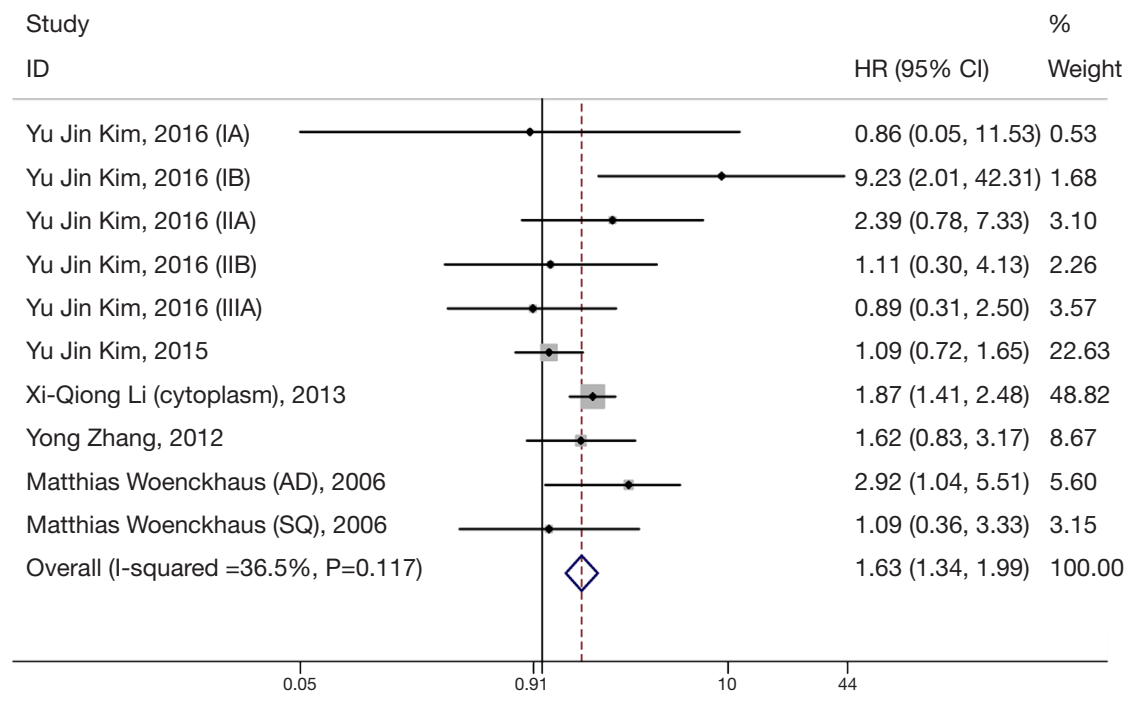

Figure 3 Meta-analysis of the association between positive cytoplasmic $\beta$-expression and prognosis in patients with non-small cell lung cancer (NSCLC). Forrest plot of hazard ratios (HRs) and 95\% confidence intervals (CI) from each study were shown.

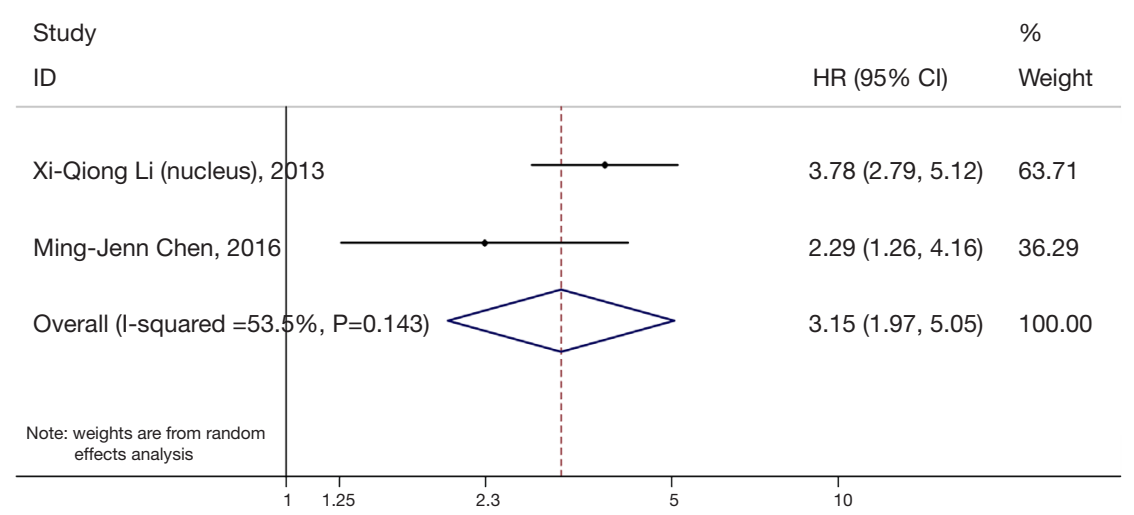

Figure 4 Meta-analysis of the association between positive nuclear $\beta$-expression and prognosis in patients with non-small cell lung cancer (NSCLC). Forrest plot of hazard ratios (HRs) and 95\% confidence intervals (CI) from each study were shown.

In the meta-analysis on cytoplasmic $\beta$-catenin expression, there was no significant heterogeneity among the studies, and subgroup analysis shown that the heterogeneity is also mild (Figure 3). On the other hand, in the meta-analysis on membranous and abnormal $\beta$-catenin expression, we found highly significant heterogeneity among the included studies when assessing the prognostic value (Figures 2,5). In the stratified analysis of the association between membranous $\beta$-catenin expression and OS, heterogeneity was not prominent in certain subgroup analyses. These findings suggest that analysis model, methods of HR estimate, the number of patients may partly account for this heterogeneity. By contrast, focusing on the studies of abnormally expressed $\beta$-catenin, we identified more significant heterogeneity after subgroup analysis based on the number of patients. Although all the studies examined the expression of $\beta$-catenin using immunohistochemical analysis, different criteria of positive aberrant $\beta$-catenin expression were made. Some only used the percentage of $\beta$-catenin expression to define positive expression, while the others used complex score multiplying percentage and intensity of $\beta$-catenin expression with various cut-off values. Therefore, no uniform standard to define the positive expression of $\beta$-catenin may cause potential bias. Besides, 


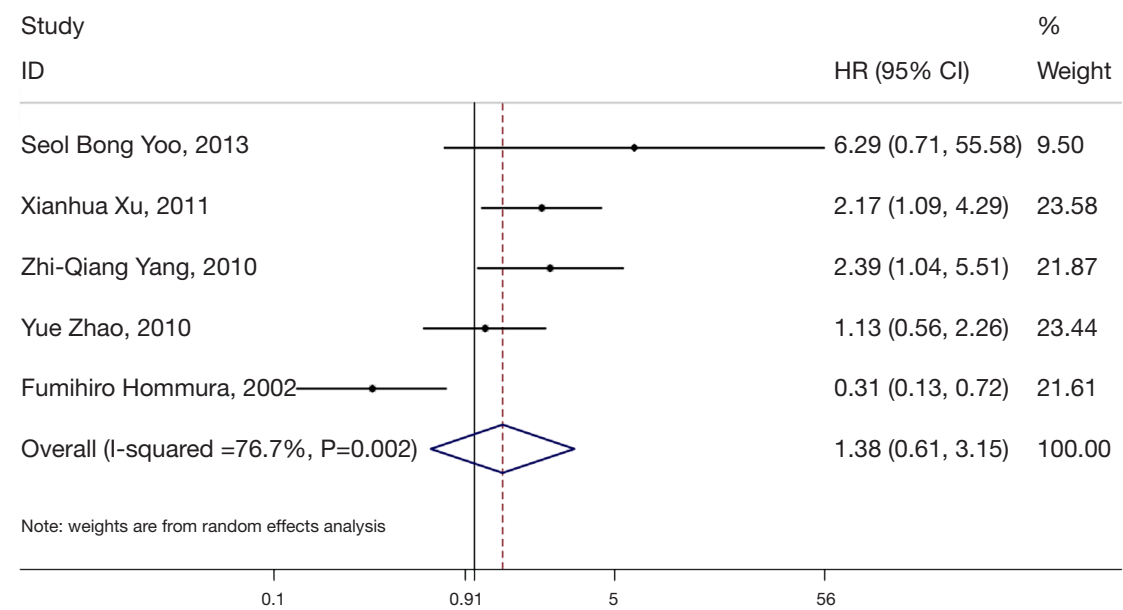

Figure 5 Meta-analysis of the association between abnormal $\beta$-expression and clinical outcomes in patients with non-small cell lung cancer (NSCLC). Forrest plot of hazard ratios (HRs) and $95 \%$ confidence intervals (CI) from each study were shown.
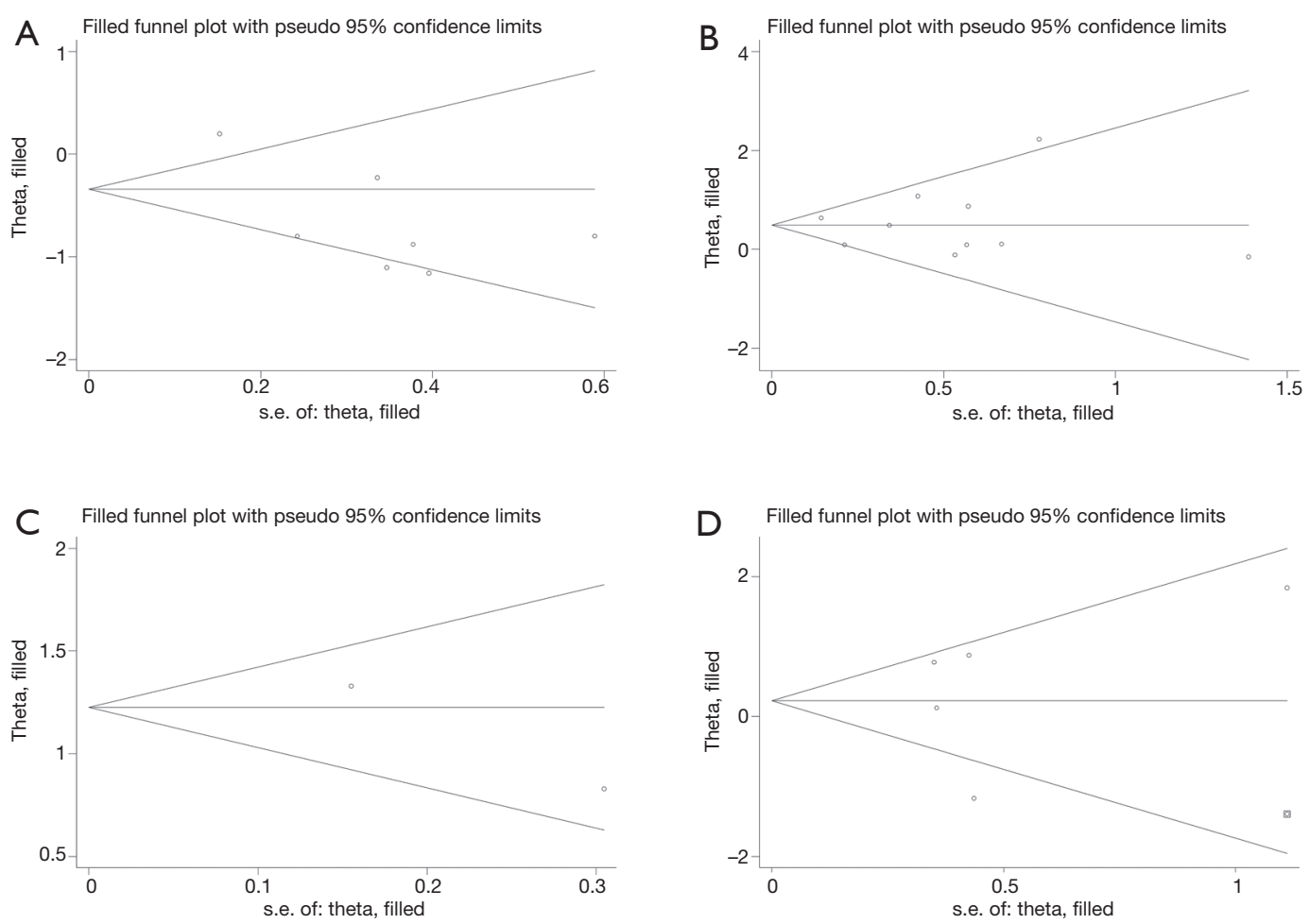

Figure 6 Begg's funnel plots for assessing potential publication bias on prognosis of patients with non-small cell lung cancer (NSCLC). (A) $\beta$-expression in membrane and overall survival; (B) $\beta$-expression in cytoplasm and survival; (C) $\beta$-expression in nucleus and overall survival; (D) $\beta$-expression in abnormal pattern and outcomes of patients. 

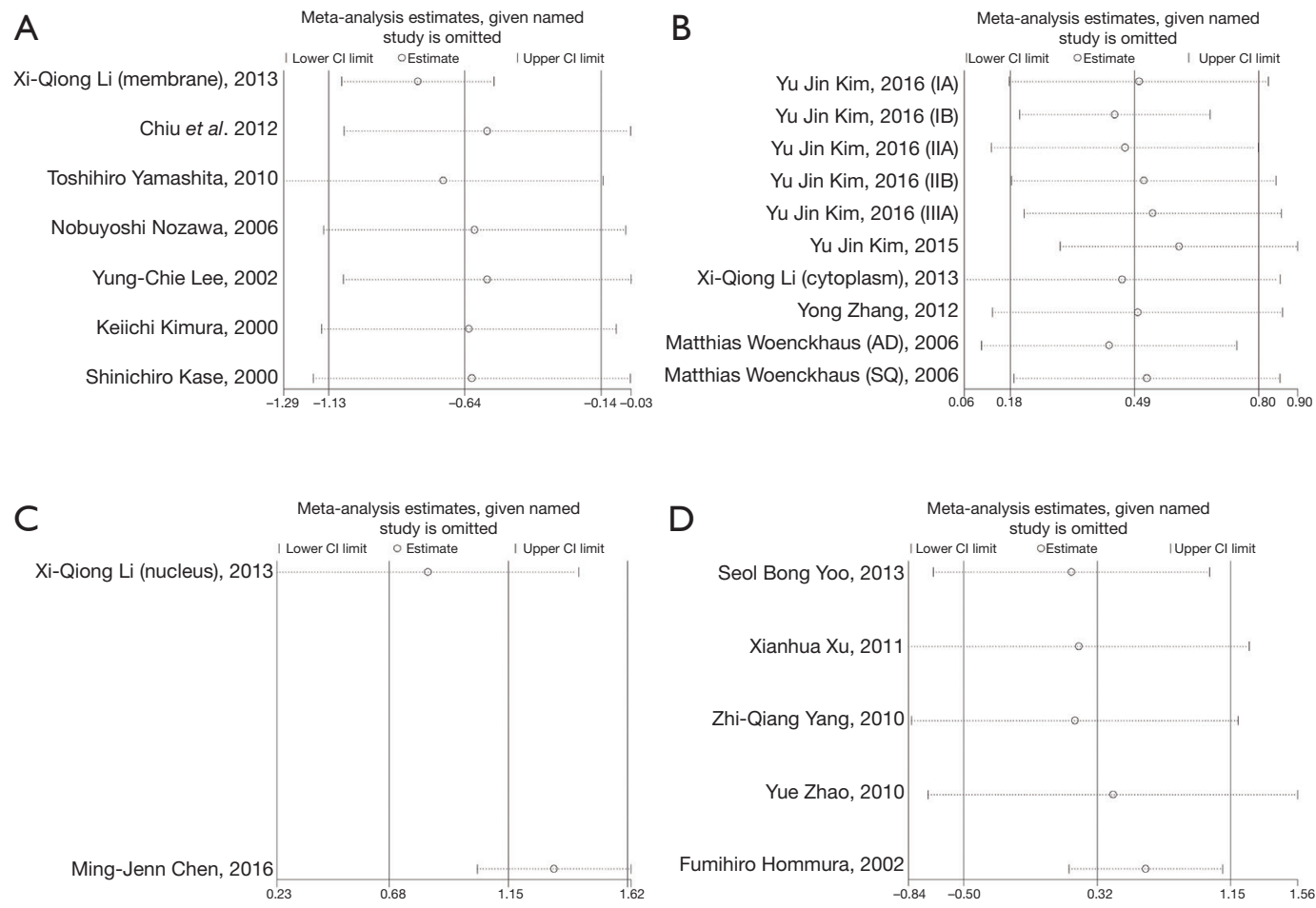

Figure 7 Sensitivity analyses for assessing heterogeneity of the meta-analysis. (A) $\beta$-expression in membrane and overall survival; (B) $\beta$-expression in cytoplasm and survival; (C) $\beta$-expression in nuclear and overall survival; (D) $\beta$-expression in abnormal pattern and outcomes of patients.

other clinical factors, such as different antibody clones used, TNM stages, age and different postoperative treatment in each study may contribute to heterogeneity in the estimate of HRs.

Sensitivity analysis also indicated that omission of any singly study did not have significant effects on the pooled risk estimates in all of the four outcomes, suggesting robust results of the meta-analysis. Moreover, Egger's tests also showed no evidence of publication in all of the four outcomes. Nevertheless, among the excluded studies, three literatures assessed the prognostic significance of $\beta$-catenin but not sufficient data was obtained. This factor could lead to possible publication bias and should not be ignored.

Mei et al. (51) has conducted a meta-analysis and suggested that reduced $\beta$-catenin expression is associated with a poor OS in NSCLC patients. But Mei et al. did not perform a subgroup analysis based on different $\beta$-catenin expression patterns. Besides, some of the studies included evaluated the influences of abnormal expression of $\beta$-catenin on OS instead of solely reduced membranous staining. Therefore, the results of our meta-analysis are more accurate and more reliable compared to the previous meta-analysis. The prognostic impacts of $\beta$-catenin on other malignant tumors were also assessed. $\mathrm{Li}$ et al. (9) revealed that aberrant expression of $\beta$-catenin, which was defined as "reduced expression in membrane" or "overexpression in cytoplasm/ nucleus", significantly influenced the survival of patients with gastric cancer (HR: 1.85; 95\% CI: 1.39-2.46). Zeng et al. (52) also suggested that altered expression of $\beta$-catenin could serve as a predicative factor of poor prognosis for patients with esophageal carcinoma. Zhang et al. (50) and Chen et al. (53) have demonstrated that positive $\beta$-catenin expression in cytoplasm/nucleus was significantly associated with poor prognosis of breast cancer and hepatocellular carcinoma respectively. Combined with our meta-analysis, these findings suggest that aberrant $\beta$-catenin expression predicts a poor survival in a wide variety of malignant tumors.

Several limitations of the present meta-analysis should be considered. First, the sample size of the included studies ranged from 35 to 576 , which could influence the precision of the results. Second, only two studies focused on nuclear $\beta$-catenin expression, which made it difficult to confirm 
the prognostic value of nuclear $\beta$-catenin for NSCLC. Third, as mentioned above, moderate and even significant heterogeneity of the analysis could be caused by several factors: different evaluation standard of protein standard, various cut-off valued and different antibody clones used.

In summary, our meta-analysis revealed that reduced membranous $\beta$-catenin immunohistochemical expression was an important prognosticator for OS. Besides, positive expression of cytoplasmic or nuclear $\beta$-catenin correlated with poor prognosis, although we did not identify a significant association between abnormal $\beta$-catenin and clinical outcome of NSCLC patients. The early detection of $\beta$-catenin may be of great value in predicting the clinical outcomes of NSCLC patients.

\section{Acknowledgements}

Funding: This study was supported by the Natural Science Foundation of Jiangsu Province (No. BK20140736), Clinical Science and Technology Project of Jiangsu Province (No. BL2013026), the National Natural Science Foundation of China (No. 81302032, No. 81401903, No. 81572937, No. 81572273), and Program of Nanjing Science and Technology of Nanjing Science and Technology Committee (No. 201605059). SS Kobayashi was supported by National Institution of Health (R21CA178301 and R01CA169259). American Cancer Society (RSG-13-047), and Harvard Stem Cell Institute Blood Program (DP-0110-12-00).

\section{Footnote}

Conflicts of Interest: The authors have no conflicts of interest to declare.

\section{References}

1. Siegel RL, Miller KD, Jemal A. Cancer statistics, 2016. CA Cancer J Clin 2016;66:7-30.

2. Zappa C, Mousa SA. Non-small cell lung cancer: current treatment and future advances. Transl Lung Cancer Res 2016;5:288-300.

3. Molina JR, Yang P, Cassivi SD, et al. Non-small cell lung cancer: epidemiology, risk factors, treatment, and survivorship. Mayo Clin Proc 2008;83:584-94.

4. Alberg AJ, Ford JG, Samet JM, et al. Epidemiology of lung cancer: ACCP evidence-based clinical practice guidelines (2nd edition). Chest 2007;132:29S-55S.

5. Donnem T, Bremnes RM, Busund LT, et al. Gene expression assays as prognostic and predictive markers in early stage non-small cell lung cancer. J Thorac Dis 2012;4:212-3.

6. Micalizzi DS, Farabaugh SM, Ford HL. Epithelialmesenchymal transition in cancer: parallels between normal development and tumor progression. J Mammary Gland Biol Neoplasia 2010;15:117-34.

7. Katoh M. Epithelial-mesenchymal transition in gastric cancer (Review). Int J Oncol 2005;27:1677-83.

8. Scanlon CS, Van Tubergen EA, Inglehart RC, et al. Biomarkers of epithelial-mesenchymal transition in squamous cell carcinoma. J Dent Res 2013;92:114-21.

9. Li LF, Wei ZJ, Sun H, et al. Abnormal beta-catenin immunohistochemical expression as a prognostic factor in gastric cancer: a meta-analysis. World J Gastroenterol 2014;20:12313-21.

10. Schmalhofer O, Brabletz S, Brabletz T. E-cadherin, betacatenin, and ZEB1 in malignant progression of cancer. Cancer Metastasis Rev 2009;28:151-66.

11. Tam WL, Weinberg RA. The epigenetics of epithelial-mesenchymal plasticity in cancer. Nat Med 2013;19:1438-49.

12. Nakayama S, Sng N, Carretero J, et al. beta-catenin contributes to lung tumor development induced by EGFR mutations. Cancer Res 2014;74:5891-902.

13. Nakata A, Yoshida R, Yamaguchi R, et al. Elevated beta-catenin pathway as a novel target for patients with resistance to EGF receptor targeting drugs. Sci Rep 2015;5:13076.

14. Pacheco-Pinedo EC, Durham AC, Stewart KM, et al. Wnt/beta-catenin signaling accelerates mouse lung tumorigenesis by imposing an embryonic distal progenitor phenotype on lung epithelium. J Clin Invest 2011;121:1935-45.

15. Kim MY, Han SI, Lim SC. Roles of cyclin-dependent kinase 8 and beta-catenin in the oncogenesis and progression of gastric adenocarcinoma. Int J Oncol 2011;38:1375-83.

16. Cheng CW, Liu YF, Yu JC, et al. Prognostic significance of cyclin D1, beta-catenin, and MTA1 in patients with invasive ductal carcinoma of the breast. Ann Surg Oncol 2012;19:4129-39.

17. Yoo SB, Kim YJ, Kim H, et al. Alteration of the E-cadherin/beta-catenin complex predicts poor response to epidermal growth factor receptor-tyrosine kinase inhibitor (EGFR-TKI) treatment. Ann Surg Oncol 2013;20 Suppl 3:S545-52.

18. Li XQ, Yang XL, Zhang G, et al. Nuclear beta-catenin 
accumulation is associated with increased expression of Nanog protein and predicts poor prognosis of non-small cell lung cancer. J Transl Med 2013;11:114.

19. Moher D, Liberati A, Tetzlaff J, et al. Preferred reporting items for systematic reviews and meta-analyses: the PRISMA statement. PLoS Med 2009;6: e1000097.

20. Phan K, Tian DH, Cao C, et al. Systematic review and meta-analysis: techniques and a guide for the academic surgeon. Ann Cardiothorac Surg 2015;4:112-22.

21. Liberati A, Altman DG, Tetzlaff J, et al. The PRISMA statement for reporting systematic reviews and metaanalyses of studies that evaluate healthcare interventions: explanation and elaboration. BMJ 2009;339:b2700.

22. Moher D, Shamseer L, Clarke M, et al. Preferred reporting items for systematic review and meta-analysis protocols (PRISMA-P) 2015 statement. Syst Rev 2015;4:1.

23. Liu Y, Yuan D, Ye W, et al. Prognostic value of circulating endothelial cells in non-small cell lung cancer patients: a systematic review and meta-analysis. Transl Lung Cancer Res 2015;4:610-8.

24. Chen MJ, Wu DW, Wang YC, et al. PAK1 confers chemoresistance and poor outcome in non-small cell lung cancer via beta-catenin-mediated stemness. Sci Rep 2016;6:34933.

25. Kim Y, Jin D, Lee BB, et al. Overexpression of betacatenin and cyclin D1 is associated with poor overall survival in stage IA-IIA squamous cell lung cancer irrespective of adjuvant chemotherapy. J Thorac Oncol 2016;11:2193-2201.

26. Kim Y, Jin D, Lee BB, et al. RARbeta2 hypermethylation is associated with poor recurrence-free survival in never-smokers with adenocarcinoma of the lung. Clin Epigenetics 2015;7:32.

27. Zhang Y, Han Y, Zheng R, et al. Expression of Frat1 correlates with expression of beta-catenin and is associated with a poor clinical outcome in human SCC and AC. Tumour Biol 2012;33:1437-44.

28. Chiu CG, Chan SK, Fang ZA, et al. Beta-catenin expression is prognostic of improved non-small cell lung cancer survival. Am J Surg 2012;203:654-9.

29. Xu X, Sun PL, Li JZ, et al. Aberrant Wnt1/beta-catenin expression is an independent poor prognostic marker of non-small cell lung cancer after surgery. J Thorac Oncol 2011;6:716-24.

30. Yamashita T, Uramoto H, Onitsuka T, et al. Association between lymphangiogenesis-/micrometastasis- and adhesion-related molecules in resected stage I NSCLC. Lung Cancer 2010;70:320-8.
31. Yang ZQ, Zhao Y, Liu Y, et al. Downregulation of HDPR1 is associated with poor prognosis and affects expression levels of p120-catenin and beta-catenin in nonsmall cell lung cancer. Mol Carcinog 2010;49:508-19.

32. Zhao Y, Yang ZQ, Wang Y, et al. Dishevelled-1 and dishevelled-3 affect cell invasion mainly through canonical and noncanonical Wnt pathway, respectively, and associate with poor prognosis in nonsmall cell lung cancer. Mol Carcinog 2010;49:760-70.

33. Woenckhaus M, Merk J, Stoehr R, et al. Prognostic value of FHIT, CTNNB1, and MUC1 expression in non-small cell lung cancer. Hum Pathol 2008;39:126-36.

34. Nozawa N, Hashimoto S, Nakashima Y, et al. Immunohistochemical alpha- and beta-catenin and E-cadherin expression and their clinicopathological significance in human lung adenocarcinoma. Pathol Res Pract 2006;202:639-50.

35. Hommura F, Furuuchi K, Yamazaki K, et al. Increased expression of beta-catenin predicts better prognosis in nonsmall cell lung carcinomas. Cancer 2002;94:752-58.

36. Lee YC, Wu CT, Chen CS, et al. The significance of E-cadherin and alpha-, beta-, and gamma-catenin expression in surgically treated non-small cell lung cancers of $3 \mathrm{~cm}$ or less in size. J Thorac Cardiovasc Surg 2002;123:502-7.

37. Kimura K, Endo Y, Yonemura Y, et al. Clinical significance of S100A4 and E-cadherin-related adhesion molecules in non-small cell lung cancer. Int J Oncol 2000;16:1125-31.

38. Kase S, Sugio K, Yamazaki K, et al. Expression of E-cadherin and beta-catenin in human non-small cell lung cancer and the clinical significance. Clin Cancer Res 2000;6:4789-96.

39. Tierney JF, Stewart LA, Ghersi D, et al. Practical methods for incorporating summary time-to-event data into metaanalysis. Trials 2007;8:16.

40. Higgins JP, Thompson SG, Deeks JJ, et al. Measuring inconsistency in meta-analyses. BMJ 2003;327:557-60.

41. Xu C, Xie D, Yu SC, et al. beta-Catenin/POU5F1/ SOX2 transcription factor complex mediates IGF-I receptor signaling and predicts poor prognosis in lung adenocarcinoma. Cancer Res 2013;73:3181-9.

42. Bleckmann A, Siam L, Klemm F, et al. Nuclear LEF1/ TCF4 correlate with poor prognosis but not with nuclear $\beta$-catenin in cerebral metastasis of lung adenocarcinomas. Clin Exp Metastasis 2013;30:471-82.

43. Miao Y, Li AL, Wang L, et al. Overexpression of NEDD9 is associated with altered expression of E-Cadherin, beta-Catenin and N-Cadherin and predictive of poor 
prognosis in non-small cell lung cancer. Pathol Oncol Res 2013;19:281-6.

44. Song DH, Ko GH, Lee JH, et al. Myoferlin expression in non-small cell lung cancer: Prognostic role and correlation with VEGFR-2 expression. Oncol Lett 2016;11:998-1006.

45. Miao Y, Wang L, Zhang X, et al. Promoter methylationmediated silencing of beta-catenin enhances invasiveness of non-small cell lung cancer and predicts adverse prognosis. PLoS One 2014;9:e112258.

46. Yang Y, Shen J, He J, et al. A meta-analysis of abnormal beta-catenin immunohistochemical expression as a prognostic factor in lung cancer: location is more important. Clin Transl Oncol 2016;18:685-92.

47. Katoh M, Katoh M. WNT signaling pathway and stem cell signaling network. Clin Cancer Res 2007;13:4042-5.

48. Königshoff M, Eickelberg O. WNT signaling in lung disease: a failure or a regeneration signal? Am J Respir Cell Mol Biol 2010;42:21-31.

49. Yang K, Wang X, Zhang H, et al. The evolving roles of

Cite this article as: Jin J, Zhan P, Katoh M, Kobayashi SS, Phan K, Qian H, Li H, Wang X, Wang X, Song Y; written on behalf of the AME Lung Cancer Collaborative Group. Prognostic significance of $\beta$-catenin expression in patients with non-small cell lung cancer: a meta-analysis. Transl Lung Cancer Res 2017;6(1):97-108. doi: 10.21037/tlcr.2017.02.07 canonical WNT signaling in stem cells and tumorigenesis: implications in targeted cancer therapies. Lab Invest 2016;96:116-36.

50. Zhang DP, Li XW, Lang JH. Prognostic Value of betacatenin Expression in Breast Cancer Patients: a Metaanalysis. Asian Pac J Cancer Prev 2015;16:5625-33.

51. Mei XD, Su H, Song J, et al. Prognostic significance of beta-catenin expression in patients with non-small cell lung cancer: a meta-analysis. Biosci Trends 2013;7:42-9.

52. Zeng R, Duan L, Kong YK, et al. Prognostic significance of beta-catenin expression in patients with esophageal carcinoma: a meta-analysis. Asian Pac J Cancer Prev 2014;15:6103-8.

53. Chen J, Liu J, Jin R, et al. Cytoplasmic and/or nuclear expression of beta-catenin correlate with poor prognosis and unfavorable clinicopathological factors in hepatocellular carcinoma: a meta-analysis. PLoS One 2014;9:e111885. 